\title{
MELHORIAS NO PROCESSO DE FLOTAÇ̃̃O POR AR DISSOLVIDO UTILIZANDO INDUÇÃO DE AR PARA PRODUÇÃO DE MICROBOLHAS
}

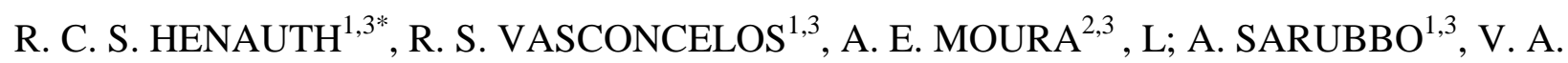 \\ SANTOS $^{1,3}$ \\ ${ }^{1}$ Universidade Católica de Pernambuco, Centro de Ciência e Tecnologia \\ ${ }^{2}$ Universidade Federal de Pernambuco, Centro de Tecnologia e Geociências \\ ${ }^{3}$ Centro de Gestão de Tecnologia e Inovação - CGTI \\ e-mail: rosangelahenauth@yahoo.com.br
}

\begin{abstract}
RESUMO
Foi desenvolvida uma técnica para a produção de microbolhas de ar, reduzindo-se espaço e custos com energia em relação ao processo convencional de saturação do efluente tratado com ar comprimido. A referida técnica foi aplicada em um protótipo de bancada de flotação por ar dissolvido (FAD), como uma etapa do tratamento de águas oleosas. Para aquisição de dados sobre a eficiência da técnica utilizada foi aplicado um delineamento composto central rotacional (DCCR). A análise desses resultados mostrou eficiência de separação acima de $90 \%$. Esse alto nível de eficiência de separação água-óleo quando comparados com valores médios da literatura evidenciam uma alta performance no que diz respeito a técnica desenvolvida.
\end{abstract}

\section{INTRODUÇÃO}

O cisalhamento causado por bombas, válvulas, constrições hidráulicas e outros equipamentos, dispersa o óleo e a água formando emulsões, que podem apresentar-se altamente estabilizadas pela presença de sólidos finamente divididos, substâncias surfactante naturais do petróleo e outros reagentes adicionados durante o processo de produção de óleos lubrificantes e combustíveis (GOBBI, 2013).

A contínua expansão da indústria de processamento de hidrocarbonetos e a extensa utilização de produtos relacionados com o petróleo aumentou a ameaça da poluição, por esse tipo de produto, ao meio ambiente. Três métodos são largamente usados para separar o óleo em suspensão e gorduras a partir de água e de águas residuárias: separação por gravidade (sedimentação), filtração e flotação por ar dissolvido (FAD) (CARTIER, 2005).

A flotação por ar dissolvido é a mais praticada porque o método é o mais versátil.

Esse processo tem despertado grande interesse para o tratamento de efluentes líquidos, especialmente aqueles efluentes em que a diferença entre as densidades de fase é pequena (CARTIER, 2005).

De modo geral, a literatura apresenta diversos trabalhos, visando o estudo de processos para a separação de emulsões água/óleo (CUNHA, 2007). Muitas técnicas para a separação dessas emulsões são disponíveis, sendo nomeadas como filtros, ultrafiltração, microfiltração, separação por gravidade, flotação por ar dissolvido, flotação em coluna, eletroflotação, flotação por ar induzido, biorreator de membrana, adsorção de carbono, coagulação química e eletrocoagulação

(SANTANDER; 
RODRIGUES; RÚBIO, 2011). Para que as fontes poluidoras possam atender às normas de lançamento estabelecidas pelas leis ambientais e reduzir os impactos gerados ao ecossistema, vários métodos de tratamento de efluentes estão disponíveis para serem empregados. Estes métodos podem ser divididos em métodos convencionais, com adição de produtos químicos e métodos alternativos, como os métodos de separação líquido-líquido (THEODORO, 2010).

Apesar de o processo FAD ter recebido uma grande atenção dos pesquisadores sobre separação água-óleo (SAO), a produção de microbolhas apresenta-se como uma etapa crítica para o processo (AZEVEDO, 2013). A utilização de equipamentos como compressor e tanques com pressurização para produção de efluente saturado, envolve consumos consideráveis de energia, precisam ser substituídos, dando lugar a equipamentos mais compactos e econômicos (SARUBBO et al., 2012a; 2012c).

Dentre as metodologias de avaliação da eficiência de um processo, a exemplo da produção de microbolhas, está a ferramenta estatística descrita como delineamento composto central rotacional. Esse modelo consiste, basicamente, na seleção de um número fixo de níveis para cada um dos fatores ou variáveis e na execução de experimentos com todas as combinações possíveis (CAPELLARI, 2010) e consta de uma parte fatorial, uma parte axial e pontos centrais (CONAGIN, 1982). Esse tipo de delineamento foi desenvolvido por Box e Wilson (1951), visando inicialmente a sua utilização em pesquisas da indústria, bem como na química. Esses pesquisadores afirmam que o delineamento composto central rotacional da superfície de resposta é o método de análise de resultados que proporciona um número maior de informações.

Neste trabalho desenvolveu-se uma técnica de produção de microbolhas sem a utilização de compressor, de forma a substituir o sistema convencionalmente utilizado.

\section{MATERIAL E MÉTODOS}

Foi construída uma unidade piloto de flotação por ar dissolvido, confeccionada em acrílico, suportada por perfis de aço carbono, para permitir a visualização de fenômenos fluidodinâmicos da distribuição do fluxo de microbolhas, apresentado na Figura 1, para tratamento de águas oleosas (SILVA et al., 2014). Essa unidade tem início em duas antecâmaras, onde, posteriormente, o fluxo do líquido a ser tratado entra em contato com o fluxo de microbolhas, as quais aderem-se às partículas de óleo dispersas. As partículas resultantes, compostas por óleo e ar, flotam por diferença de densidade com a fase aquosa. Pela parte superior de cada uma das câmaras de flotação são retiradas as partículas oleosas em forma de espuma e, pela parte inferior, dessas câmaras, são coletadas as águas tratadas. Uma bomba centrífuga coleta a água tratada recolhida da segunda câmara descartando a mesma para reuso ou tratamento auxiliar. Uma segunda bomba é usada especificamente para produção de microbolhas, recirculando uma outra parte dessa água tratada. Para obtenção dessas microbolhas, uma quantidade adequada de ar é simultaneamente succionada na linha de aspiração dessa bomba, misturando-se com a água e saturando a mesma com auxílio do aumento de pressão na linha de descarga. A tubulação de transporte dessa água saturada por ar é seccionada em duas partes e, com auxílio de uma válvula instalada anterior a essa bifurcação, dá origem a dois fluxos de microbolhas, de mesma intensidade, um para cada câmara. 
Figura 1- Esquema 3D da unidade piloto de flotação por ar dissolvido utilizada neste trabalho

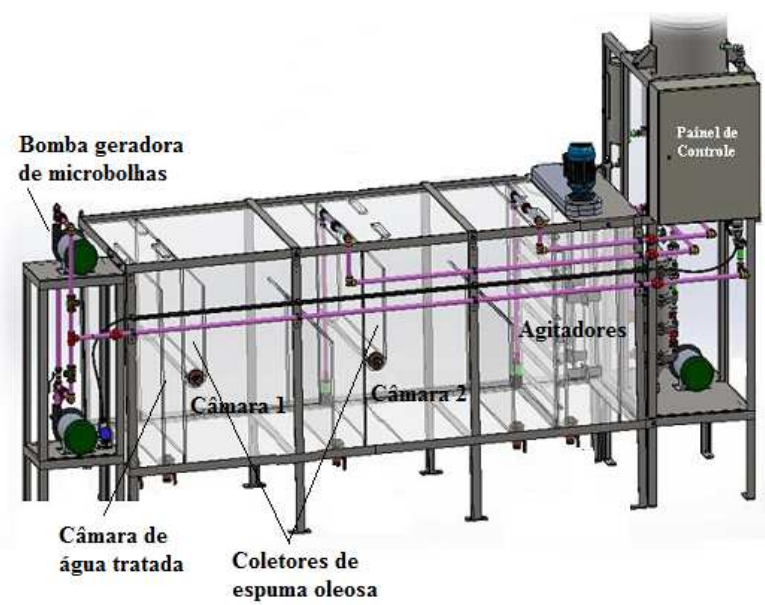

Fonte: Henauth, 2015

Para avaliação da eficiência de produção das microbolhas, foi aplicado um delineamento composto central rotacional. As variáveis estudadas foram pressão manométrica $\left(X_{1}\right)$ e a razão entre as vazões de ar/água $\left(X_{2}\right)$. Na Tabela 1 são apresentadas as faixas de valores reais $\mathrm{e}$ codificados utilizados para os ensaios.

Tabela 1 - Faixas de valores reais e codificados utilizados no DCCR

\begin{tabular}{lccccc}
\hline \multicolumn{7}{c}{ Nível } & & & \\
\hline Fatores & $-1,41$ & $-1,0$ & 0 & $+1,0$ & $+1,41$ \\
$X_{1}$ (bar) & 1,186 & 1,6 & 2,6 & 3,6 & 1,336 \\
$X_{2}\left(1: 10^{3}\right)$ & 0,234 & 0,4 & 0,8 & 1,2 & 4,914 \\
\hline
\end{tabular}

Fonte: Henauth, 2015

Dessa forma, foi realizado um total de 12 experimentos: 4 pontos fatoriais completos, 4 pontos centrais e 4 pontos axiais, sendo os mesmos executados em sequência aleatória conforme em uma matriz de planejamento. As distâncias dos pontos axiais foram $\pm 1,41$.

\section{RESULTADOS E DISCUSSÃO}

Os valores obtidos na Tabela 1 geraram os resultados de ensaios preliminares, conforme apresentados na Tabela 2. Os valores de máxima eficiência encontram-se nos pontos centrais, demonstrando um acerto referente aos valores dos experimentos realizados.

Tabela 2 - Delineamento Composto Central Rotacional com variáveis independentes em valores codificados e reais e variável dependente com valores experimentais

\begin{tabular}{ccccc}
\hline \multicolumn{2}{c}{$\begin{array}{c}\text { Valores } \\
\text { Codificados }\end{array}$} & \multicolumn{2}{c}{$\begin{array}{c}\text { Valores } \\
\text { Reais }\end{array}$} & $\begin{array}{c}\text { Valores } \\
\text { Experimentais }\end{array}$ \\
\hline $\mathrm{X}_{1}$ & $\mathrm{X}_{2}$ & $\mathrm{X}_{1}$ & $\mathrm{X}_{2}$ & $\mathrm{Y}$ \\
-1 & -1 & 1,600 & 0,400 & 23 \\
-1 & 1 & 1,600 & 1,200 & 70 \\
1 & -1 & 3,600 & 0,400 & 26 \\
1 & 1 & 3,600 & 1,200 & 59 \\
1,414 & 0 & 1,185 & 0,800 & 44 \\
1,414 & 0 & 4,014 & 0,800 & 25 \\
0 & 1,414 & 2,600 & 0,234 & 15 \\
0 & 1,414 & 2,600 & 1,365 & 62 \\
0 & 0 & 2,600 & 0,800 & 75 \\
0 & 0 & 2,600 & 0,800 & 77 \\
0 & 0 & 2,600 & 0,800 & 78 \\
0 & 0 & 2,600 & 0,800 & 74 \\
\hline
\end{tabular}

Fonte: Henauth, 2015

O diagrama de Pareto, apresentado pela Figura 2, os fatores que ultrapassam a linha pontilhada representam as variáveis com efeito significante sobre a variável resposta com $95 \%$ de confiança. A partir desse gráfico observa-se que todas as variáveis são, estatisticamente, significantivas, com destaque para o valor positivo do termo linear da razão entre as vazões ar/água e valores negativos dos demais termos. Isso demonstra que os demais termos têm valores críticos, acima dos quais a eficiência de separação água/óleo diminui. Por outro lado, a interação entre os fatores mostrou-se pouco significativa, de modo a se poder variar qualquer um deles para se obter um efeito desejado sem 
interferência na contribuição do outro fator.

Figura 2 - Diagrama de Pareto

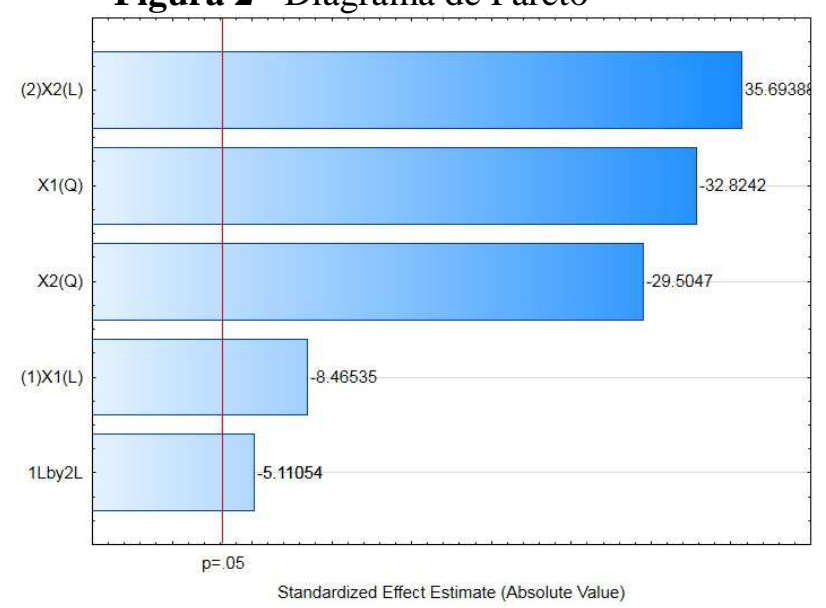

Fonte: Henauth, 2015

O modelo de previsão obtido para a eficiência de produção de microbolhas $(Y)$, recebeu uma variância explicada, ou $96 \%$ de explicação para as variações no fenômeno estudado. A Equação 1 apresenta o modelo de previsão obtido:

$$
\begin{aligned}
& Y=-287+209 \cdot X_{1}-117 \cdot X_{1}{ }^{2}+144 \cdot X_{2} \\
& -17 \cdot X_{2}{ }^{2}-9 \cdot X_{1} X_{2}
\end{aligned}
$$

Como uma quantidade maior de microbolhas produzida resulta em um número maior de gotas de óleo envolvidas e flotadas, associou-se a eficiência de produção de microbolhas à eficiência de separação água/óleo. A superfície de resposta mostrada na Figura 3, obtida com auxílio da Equação 1 ilustra a dependência da eficiência de produção de microbolhas com a pressão manométrica $\left(X_{1}\right)$ e a razão entre as vazões ar/água $\left(X_{2}\right)$. Valores experimentais desses dois fatores da ordem de 3,8 bar e 1:1000, respectivamente originaram o valor máximo para eficiência de produção de microbolhas.

Como uma quantidade maior de microbolhas produzida resulta em um número maior de gotas de óleo envolvidas e flotadas. A superfície de resposta mostrada na Figura 3, obtida com auxílio da Equação 1, ilustra a dependência da eficiência de separação, associada à eficiência de produção de microbolhas, sob a dependência dos fatores: pressão manométrica $\left(\mathrm{X}_{1}\right)$ e a razão entre as vazões ar/água $\left(\mathrm{X}_{2}\right)$.

Figura 3 - Eficiência de produção de microbolhas em função das variáveis independentes

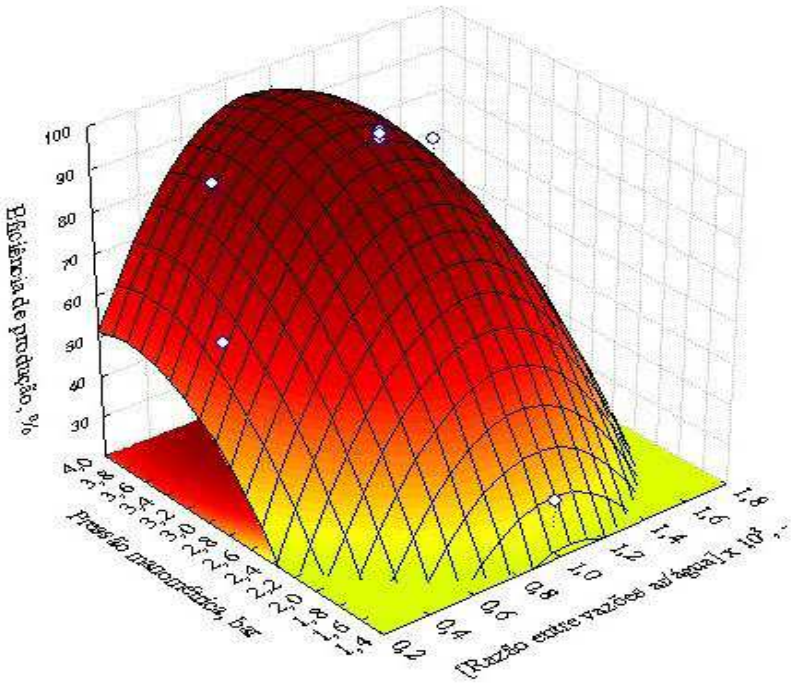

Fonte: Henauth, 2015

\section{CONCLUSÕES}

A utilização de uma bomba centrífuga com entrada de ar controlada, para dentro do tubo de sucção, deixou de ser estritamente uma preocupação com as condições operacionais desse equipamento e provou ser uma estratégia eficaz para a produção de microbolhas a partir do ar atmosférico.

O sucesso do método de produção de microbolhas descrito neste trabalho constitui uma melhoria na flotação por ar dissolvido. Este método de separação gravitacional pode ser expresso pelo método FAD modificado e é vantajoso tanto em termos técnicos (redução nas peças de equipamentos necessários) quanto em termos econômicos (redução em equipamentos e custos de energia). Futuros estudos devem ser realizados com este método usando substâncias 
auxiliares de floculação (surfactantes) e sua aplicação em diferentes processos de separação, tais como flutuação coluna, lagoas de estabilização, wetlands construídos, etc

\section{NOMENCLATURA}

$\mathrm{X}_{1}=$ Pressão manométrica (bar);

$\mathrm{X}_{2}=$ Razão entre as vazões ar/água (adimensional);

$\mathrm{Y}=$ Eficiência de separação (\%)

\section{REFERÊNCIAS}

AZEVEDO, A. C. Estudo de flotação por ar dissolvido com a bomba multifásica (FAD-B) e sedimentação lamelar (SL) no tratamento de água bruta para abastecimento público. 2012. 157p. Dissertação (Mestrado em Engenharia) Universidade Federal do Rio Grande do Sul, Canoas, 2013.

BOX, G. E. P.; WILSON, K. B. On The Experimental Attainment of Optimum Conditions. J. R. Stat. Soc., Ser. B (Statistical Methodology), Manchester, v.13, n.1, p.1-38, 1951.

CAPELLARI, J. B. Biossíntese de Ácido Láctico por Lactobacillus amylovorus a Partir de Resíduos Agroindustriais. 2010. 67p. Dissertação (Mestrado em Engenharia de Processos) - Universidade da Região de Joinville - UNIVILLE, Joinville, 2010.

CARTIER, M. L. M. Desenvolvimento de um sensor para monitoramento "on-line" da contaminação da água por hidrocarbonetos. 2005. 72p. Iniciação Científica (Universidade Federal de Itajubá) - Programa de Recursos Humanos da ANP Para o Setor de Petróleo e Gás - PRHANP/MME/MCT, Itajubá/MG. 2005.

CONAGIN, A. Delineamento Composto Central com Duas Estrelas. Pesq. Agropec. Bras. v.17, n.9, p.1261- 9, 1982.

CUNHA, R. E. P. Modelagem matemática da separação gravitacional de emulsões de petróleo. 2007. 136p. Dissertação (Mestrado em Engenharia de Processos) - Universidade Tiradentes, Aracaju, 2007.

GOBBI, L. C. A. Tratamento de água oleosa por eletrofloculação. 2013. 120p. Dissertação (Mestrado em Energia) - Universidade Federal do Espírito Santo, São Mateus, 2013.

HENAUTH, R. C. S. Produção de Microbolhas Utilizando a Indução de Ar Atmosférico para Melhorias no Processo de Flotação Por Ar Dissolvido (FAD). 2014. 101p. Dissertação (Mestrado em Desenvolvimento de Processos Ambientais) - Universidade Católica de Pernambuco, Recife, 2015.

SANTANDER, M.; RODRIGUES, R. T.; RUBIO, J. Modified jet flotation in oil (petroleum) emulsion/water separations. Colloids And Surfaces A: Physicochem. Eng. Aspects, v.375, p.237-244, 2011.

SARUBBO, L. A.; LUNA, J. M.; RUFINO, R. D.; FARIAS, C. B. B.; SANTOS, V. A. Production of biosurfactants for application in the removal of hydrophobic contaminants originated by the petroleum industry In: CHEMICAL ENGINEERING TRANSACTIONS, Milan. 5th International Conference on Safety and Environmental in Process \& Power Industry. Milan: The Italian Association of Chemical Engineering, v.27, 2012a.

SARUBBO, L. A.; RUFINO, R. D.; LUNA, J. M.; FARIAS, C. B. B.; SANTOS, V. A. Utilização de superfície de resposta para otimização da produção de um biossurfactante com aplicação na remoção de derivados de petróleo In: RIO OIL \& GAS EXPO AND CONFERENCE, 2012, Rio de Janeiro. Rio oil \& gas 30 years. resumos... Rio de Janeiro: ibp, 
2012c.

SILVA, F.C.P.R.E., SILVA, N.M.P.R., MOURA, A.E., ALMEIDA, R.G., LUNA, J.M., RUFINO, R.D., SANTOS, V.A., SARUBBO, L.A. Effect of biosurfactant addition in a pilot scale dissolved air flotation system. Separ. Sci. Technol., 2014.

THEODORO, P. S. Utilização da eletrocoagulação no tratamento de efluentes na indústria galvânica. 2010. 112p. Dissertação (Mestrado em Engenharia Química) Universidade Estadual do Oeste do Paraná, Toledo, 2010.

\section{AGRADECIMENTOS}

À FACEPE, pela concessão da bolsa de Mestrado, à UNICAP, pela oportunidade de realização do Mestrado, ao Centro de Gestão de Tecnologia e Inovação (CGTI) e a Candeias Energia S/A, pelo apoio técnico concedido. 